\title{
Review
}

Journal of Innate

Immunity
J Innate Immun 2009;1:176-180

DOI: $\underline{10.1159 / 000203699}$
Received: October 3, 2008

Accepted after revision: October 16, 2008

Published online: February 20, 2009

\section{Neutrophil Extracellular Traps: A Strategic Tactic to Defeat Pathogens with Potential Consequences for the Host}

\section{Eva Medina}

Infection Immunology Research Group, Department of Microbial Pathogenesis, HZI - Helmholtz Center for Infection Research, Braunschweig, Germany

\section{Key Words}

Neutrophils - Neutrophil extracellular traps • Pathogens · Antimicrobial mechanisms - Mast cell extracellular traps · ETosis $\cdot$ Reactive oxygen radicals

\begin{abstract}
Recent investigations have highlighted new roles for neutrophils in the biology of infection and inflammation. Neutrophils are one of the main players in the innate immune system and actively contribute to host defense by killing pathogens. Added to their ability to eliminate microorganisms by phagocytosis, neutrophils can also kill microbes by capturing them in extracellular structures consisting of granule proteins and DNA called neutrophil extracellular traps (NETs). This review summarizes the recent advancements regarding the structure, production and biological relevance of NETs.

Copyright $\odot 2009$ S. Karger AG, Basel
\end{abstract}

\section{Introduction}

Due to their ability to kill pathogens, neutrophils have long been recognized as one of the main cellular components of the innate immune response against infectious microorganisms. Neutrophils are terminally differenti- ated cells when they leave the bone marrow and they circulate for a brief period of time before migrating into tissues [1]. Upon microbial challenge, neutrophils are one of the first immune cells recruited to an infection site where they can defeat the insidious pathogen by using different mechanisms. The classical way consists of engulfing and killing the internalized pathogens by exposing them to oxidative burst and antimicrobial compounds discharged into the phagocytic vacuole by cytoplasmic granules [2, 3]. More recently, indisputable evidence has been provided that neutrophils can kill pathogens in an extracellular manner, which does not require phagocytic uptake [4]. This novel mechanism, described for the first time by Brinkmann et al. [4], consists of the release of web-like structures of DNA and proteins (neutrophil extracellular traps, NETs) by activated neutrophils that bind, disarm and kill pathogens extracellularly. The field of innate immunity has been, therefore, enriched by the description of this fascinating extracellular antimicrobial weapon of neutrophils.

\section{The Structure of NETs}

NETs are formed upon activation of neutrophils with phorbol myristate acetate (PMA), interleukin-8 (IL-8), lipopolysaccharide (LPS) or pathogens such as Shigella

\section{KARGER}

Fax +4161306 1234

E-Mail karger@karger.ch

www.karger.com
(C) 2009 S. Karger AG, Basel

$1662-811 X / 09 / 0013-0176 \$ 26.00 / 0$

Accessible online at:

www.karger.com/jin
Dr. Eva Medina

Infection Immunology Research Group, Department of Microbial Pathogenesis Helmholtz Center for Infection Research

Inhoffenstrasse 7, DE-38124 Braunschweig (Germany)

Tel. +495316181 4500, Fax +495316181 4499, E-Mail eva.medina@helmholtz-hzi.de 

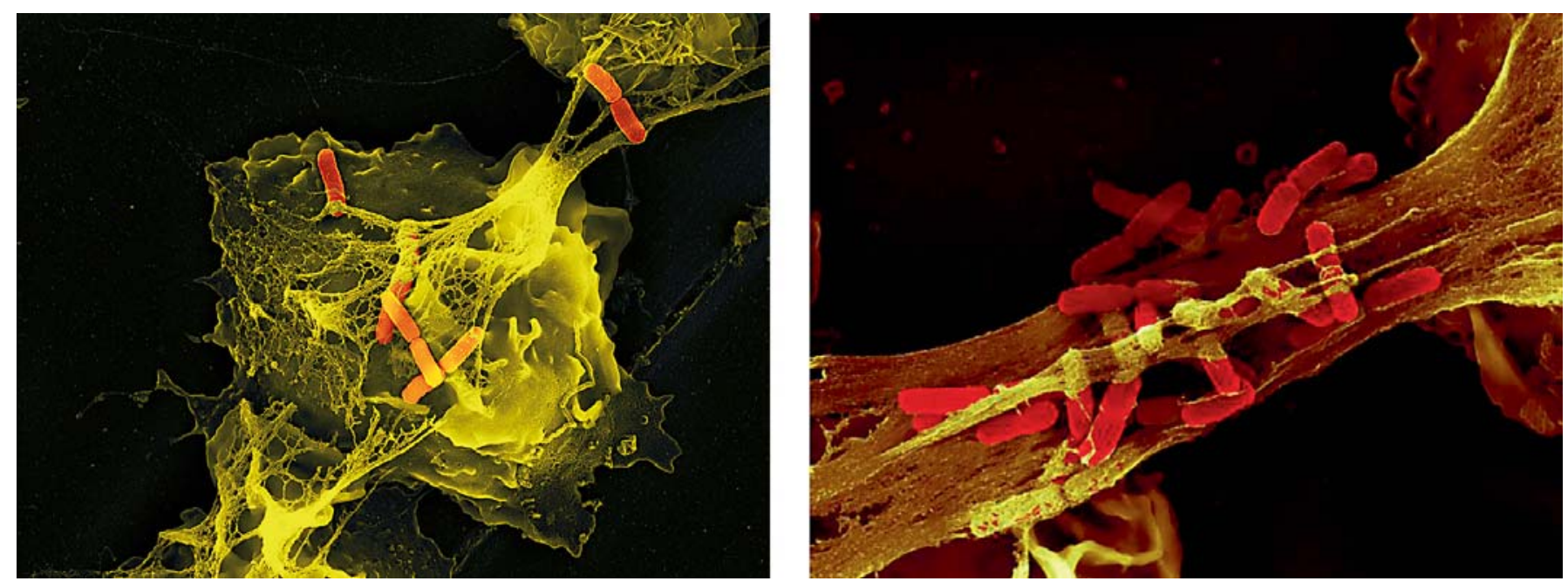

Fig. 1. Colored scanning electron photographs showing Shigella flexneri microorganisms (red) entrapped by NETs. Provided by V. Brinkmann, Department of Cellular Microbiology, Max Planck Institute for Infection Biology, Berlin, Germany.
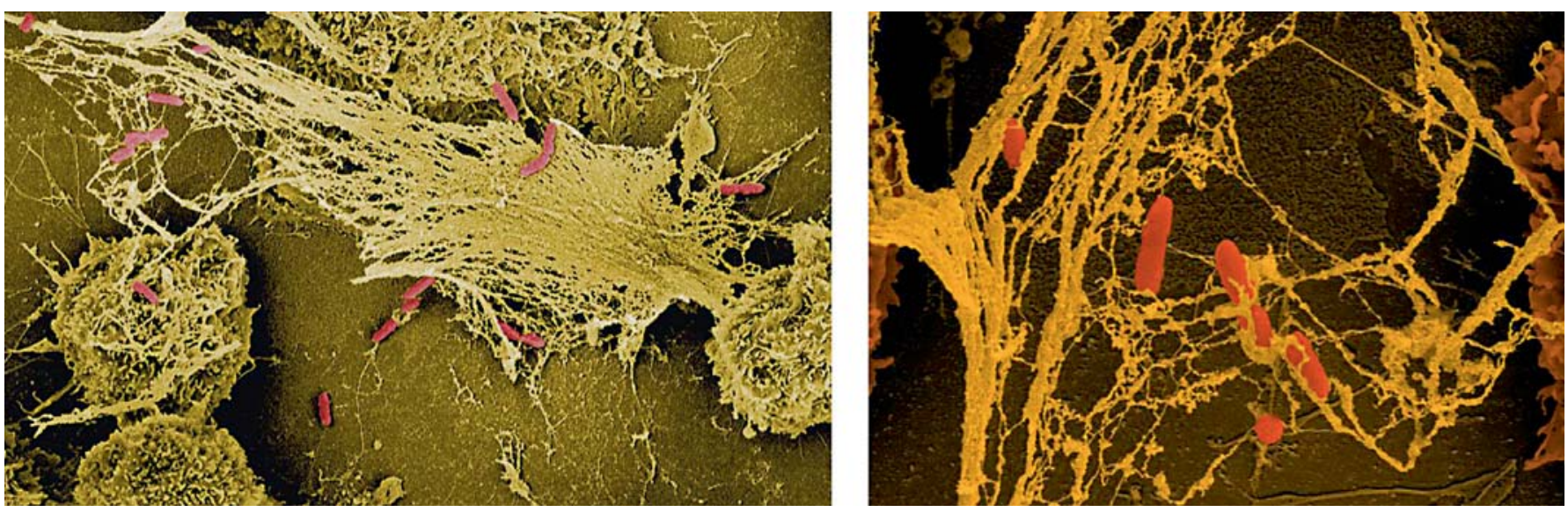

Fig. 2. Colored scanning electron photographs showing Pseudomonas aeruginosa microorganisms (red) entrapped by mast cell extracellular traps (MCETs). Provided by M. Rohde, Department of Microbial Pathogenesis, Helmholtz Center for Infection Research, Braunschweig, Germany.

flexneri, Staphylococcus aureus, Salmonella typhimurium, Streptococcus pneumoniae, Streptococcus pyogenes and the fungus Candida albicans. NETs consist of smooth fibers with a diameter of $15-17 \mathrm{~nm}$, which are probably comprised of a sequence of nucleosomes from unfolded chromatin and globular domains of 25-28 $\mathrm{nm}$ diameter [4]. The interweave of these chromatin strands results in an extracellular three-dimensional structure surrounding the neutrophil from where they emerge (fig. 1). The major constituent of these fibers is DNA with embedded granular antimicrobial peptides and enzymes such as elastase, cathepsin G, and myeloperoxidase [4]. Additional component of the NETs are histones $\mathrm{H} 1, \mathrm{H} 2 \mathrm{~A}, \mathrm{H} 2 \mathrm{~B}$, $\mathrm{H} 3$, and $\mathrm{H} 4$ [4]. DNA is the main structural component and scaffold of NETs, since treatment with deoxyribonuclease (DNase) but not with proteases results in the disintegration of these structures [4]. Interestingly, cytoplasmic proteins such as actin and tubulin are excluded from the NETs [4]. 


\section{The Genesis of NETs}

How neutrophils generate and release the NETs has been one of the most enigmatic aspects of this antimicrobial mechanism. NETs are released upon activation by PMA, IL-8, LPS, bacteria and fungi. Nevertheless, stimulation of neutrophils with microorganisms seems to be much more efficient at inducing NET formation than stimulation with single bacterial components such as LPS $[5,6]$, suggesting a requirement for multiple signaling to achieve optimal NETs production by neutrophils.

NETs are released by dying neutrophils after the rupture of the cell membrane. However, formation of NETs is not the result of leakage during necrotic cell death as shown by a lack of detection of the cytoplasmic marker lactate dehydrogenase as well as by the exclusion of vital dyes by activated neutrophils for at least $2 \mathrm{~h}$ after stimulation $[5,6]$. The process of NET formation is also clearly distinct from apoptosis, since it occurs as early as $10 \mathrm{~min}$ after activation, too rapidly to be caused by apoptosis [5, 6]. In addition, NET-forming neutrophils lack the DNA fragmentation and exposure of phosphatidylserine typical of the apoptotic cell death pathway [7]. Therefore, NET formation seems to be the consequence of a novel form of active cell death that is neither necrosis nor apoptosis. Several of the features of this new death cell pathway, which has been termed ETosis [8], have been recently elucidated by Fuchs et al. [6]. During NET formation the nuclei of neutrophils lose their shape and the eu- and heterochromatin homogenize. Mixing of the NET components takes place as the nuclear envelope and the granule membranes disintegrate and nuclear material comes into direct contact with granule components. In a final step, the cell membrane breaks and the NETs are released concomitant with cell death [6].

Several lines of evidence have demonstrated that the generation of reactive oxygen species (ROS) by NADPH oxidase is required for the formation of NETs. Thus, pharmacological inhibition of the respiratory burst after treatment of neutrophils with diphenylene iodonium strongly impaired NET formation [6]. In addition, exogenous $\mathrm{H}_{2} \mathrm{O}_{2}$ has been shown to be a potent inductor of NETs, while exogenous catalase, which degrades $\mathrm{H}_{2} \mathrm{O}_{2}$, impeded the formation of NETs [6]. Finally, neutrophils isolated from patients with chronic granulomatous disease, which carry mutations in NADPH oxidase, failed to produce NETs after stimulation with PMA but not after stimulation with $\mathrm{H}_{2} \mathrm{O}_{2}$ [6]. Although the role of ROS as mediators of NETs formation is undeniable, how ROS induce ETosis cell death of neutrophils and the signaling cascade involved remain unclear. The possibility that ROS can act as a second messenger promoting the downstream signaling events leading to ETosis and NET formation deserve further investigations.

\section{The Antimicrobial Effect of NETs}

During the infection process, neutrophils will actively contribute to kill pathogens by both phagocytosis and production of NETs. NETs have been shown to entrap and disarm a wide range of bacterial pathogens such as S. flexneri, S. aureus, S. typhimurium, S. pneumoniae and $S$. pyogenes, but also pathogenic fungi such as C. albicans (fig. 1) [4, 9-11]. Although the molecular mechanism underlying the entrapment of microorganisms by the NETs is unclear, electrostatic interactions between cationic NETs components and the negatively charged surface of microorganisms has been proposed as potential mechanism. Intact NET structure is crucial for effective bactericidal activity, since negligible pathogen killing can be observed when NETs are dismantled after treatment with DNase [4]. Pathogens entrapped in the NETs are killed possibly after being exposed to a high local concentration of antimicrobial proteins such as neutrophil elastase, bactericidal permeability-increasing protein and histones [4].

Although most pathogens are killed after they have been trapped by NETs [4, 9], some bacteria have developed strategies to escape trapping and killing. One such mechanism includes the ability to produce DNases found in several pathogenic bacteria such as $S$. pyogenes, $S$. aureus or S. pneumonia [12-14]. By producing DNases, these pathogens can escape the NETs by degrading the DNA backbone [10, 11]. Thus, Buchanan et al. [11] have demonstrated that expression of DNase Sdal is an important virulence attribute of $S$. pyogenes, since DNase-deficient strains are more sensitive to extracellular neutrophil killing in vitro than those actively producing the nuclease. In addition, transformation of nonpathogenic Lactococcus lactis with a plasmid containing the Sdal gene enhanced the bacteria resistance to extracellular killing by neutrophils [11]. The relevance of Sdal during in vivo infection was demonstrated by significantly larger lesions developed in mice after subcutaneous challenge with $S$. pyogenes expressing DNase than in those challenged with a DNase-deficient strain [11].

Also S. pneumoniae expresses a surface nuclease (endA) that enables the bacterium to degrade the DNA scaffold of NETs and to escape [10]. A mutant lacking endA infects the upper respiratory tract in mice but fails 
to disseminate into the lungs and bloodstream [10]. In addition, the expression of capsule enables $S$. pneumoniae to reduce the binding and entrapments of NETs [15].

\section{The Role of NETs in Host Defense}

One crucial issue is the relevance of NET formation in in vivo conditions. NETs have been found to be abundant at sites of infection. NETs have been observed in samples from experimental shigellosis in rabbits as well as in spontaneous appendicitis in humans [4]. NETs have also been described lining the alveoli in the lungs of mice infected with S. pneumoniae [10] as well as at the skin infection foci in a murine model of $S$. pyogenes-induced necrotizing fasciitis [11]. Recently, NETs were also found in bovine mastitis [16].

In addition to their antimicrobial properties, the confinement of pathogens to a local site of infection might be an important function of NETs. Thus, NETs may serve as a physical barrier that prevents further spread of bacteria. In addition, immobilizing neutrophil granule components into NETs may keep potentially noxious proteins like proteases from diffusing away and inducing damage in tissue adjacent to the site of inflammation.

\section{The Contribution of NETs to Pathological Conditions}

Production of NETs might also have a deleterious effect on the host due to the release of high levels of DNA and histones which could play a role during the development of autoimmune diseases like lupus erythematosus, induced by autoimmune reactions to the host's own DNA [17].

NETs might also be involved in the pathogenesis of human preeclampsia, a leading cause of fetal and maternal mortality worldwide [18]. Preeclampsia is a severe disorder of late pregnancy characterized by neutrophil activation and increased levels of cell-free DNA in the maternal plasma $[19,20]$. As placenta-derived syncytiotrophoblast microparticles have been reported to be capable to induce NET formation in vitro [21], it was proposed that a massive NET induction by the placentally derived factors (IL-8 and placental microdebris) might be involved in the pathogenesis of preeclampsia [21]. Increased presence of NETs in preeclampsia can explain the increased levels of maternal circulatory cell-free DNA and might play a role in the deficient placental perfusion associated with this disorder.
It has also been reported that under extreme conditions such as severe sepsis, platelets can induce activation of neutrophils sequestered in the microvascular bed in a Toll-like receptor 4-dependent manner, leading to the production of NETs [22]. The NETs seem to retain their integrity under flow conditions and ensnare bacteria within the vasculature at the expense of injury to endothelium and tissues. Tissue injury is probably caused by the reduction in sinusoidal perfusion of blood, causing ischemic conditions, as well as by the release of proteases into the extracellular vascular compartment.

\section{Production of Extracellular Traps by Innate Immune Cells Other than Neutrophils}

Recently, it has been reported that mast cells can kill bacteria by entrapping them in extracellular structures similar to the NETs [23]. Mast cells are tissue cells that are located preferentially at the host-environment interface. After activation, they exert their biological effects by releasing preformed and de novo-synthesized mediators such as histamine, proteases, leukotrienes, prostaglandins and various cytokines [24]. Mast cells can mediate bacterial clearance at sites of infection through the release of TNF- $\alpha$ and recruitment of neutrophils $[25,26]$. In addition, mast cells themselves can also directly kill pathogens either by classical phagocytosis or by entrapping them in extracellular structures similar to NETs, which have been termed MCETs (fig. 2) [23-26]. The MCETs are produced by mast cells in response to either pathogens or PMA stimulation and are composed by DNA, histones and granule proteins such as tryptase and the antimicrobial peptide LL-37 [23]. As shown with neutrophils, disruption of MCETs results in a significant reduction of the extracellular antimicrobial effect of mast cells, suggesting that intact extracellular webs are critical for effective inhibition of bacterial growth [23]. Similar to NETs, production of MCETs is mediated by ROS-dependent cell death accompanied by disruption of the nuclear envelope, which can be induced after stimulation of mast cells with PMA, $\mathrm{H}_{2} \mathrm{O}_{2}$ or bacterial pathogens [23]. Mast cells are, therefore, the first innate cell population other than neutrophils reported to generate antimicrobial extracellular traps. Whether other cells of the innate immune system are also capable of producing these antimicrobial structures requires further investigations. 


\section{Conclusions}

During the past years, significant efforts have been made to dissect the molecular mechanisms governing the generation of NETs. Nevertheless, more work has to be done to understand the mechanism of pathogen trapping within NETs. In addition, the downstream mechanisms, specifically how ROS triggers cell death, still remain to be characterized.

The biological significance of NETs is just beginning to be explored. Further studies are required to investigate the contribution of NETs to other inflammatory and au- toimmune disorders where neutrophil activation has been shown. This might be the future challenge for the next years in NETs research and this will presumably open new avenues for the development of tools to modulate inflammation.

\section{Acknowledgments}

The author would like to thank Dr. V. Brinkmann and Dr. M. Rohde for kindly providing the electron micrographs.

\section{References}

1 Nathan C: Neutrophils and immunity: challenges and opportunities. Nat Rev Immunol 2006;6:173-182.

$\checkmark 2$ Hampton MB, Kettle AJ, Winterbourn CC: Inside the neutrophil phagosome: oxidants, myeloperoxidase, and bacterial killing. Blood 1998;92:3007-3017.

-3 Segal AW: How neutrophils kill microbes. Annu Rev Immunol 2005;23:197-223.

-4 Brinkmann V, Reichard U, Goosmann C, Fauler B, Uhlemann Y, Weiss DS, Weinrauch Y, Zychlinsky A: Neutrophil extracellular traps kill bacteria. Science 2004;303:15321535.

5 Brinkmann V, Zychlinsky A: Beneficial suicide: why neutrophils die to make NETs. Nat Rev Microbiol 2007;5:577-582.

-6 Fuchs TA, Abed U, Goosmann C, Hurwitz R, Schulze I, Wahn V, Weinrauch Y, Brinkmann V, Zychlinsky A : Novel cell death program leads to neutrophil extracellular traps. J Cell Biol 2007; 176:231-241.

7 Fink SL, Cookson BT: Apoptosis, pyroptosis, and necrosis: mechanistic description of dead and dying eukaryotic cells. Infect Immun 2005;73:1907-1916.

8 Wartha F, Henriques-Normark B: ETosis: a novel cell death pathway. Sci Signal 2008;1: pe25.

-9 Urban CF, Reichard U, Brinkmann V, Zychlinsky A: Neutrophil extracellular traps capture and kill Candida albicans yeast and hyphal forms. Cell Microbiol 2006;8:668-676.

10 Beiter K, Wartha F, Albiger B, Normark S, Zychlinsky A, Henriques-Normark B: An endonuclease allows Streptococcus pneumoniae to escape from neutrophil extracellular traps. Curr Biol 2006;16:401-407.
11 Buchanan JT, Simpson AJ, Aziz RK, Liu GY, Kristian SA, Kotb M, Feramisco J, Nizet V: DNase expression allows the pathogen group A Streptococcus to escape killing in neutrophil extracellular traps. Curr Biol 2006;16: 396-400.

12 Sumby P, Barbian KD, Gardner DJ, Whitney AR, Welty DM, Long RD, Bailey JR, Parnell MJ, Hoe NP, Adams GG, Deleo FR, Musser JM: Extracellular deoxyribonuclease made by group A Streptococcus assists pathogenesis by enhancing evasion of the innate immune response. Proc Natl Acad Sci USA 2005;102:1679-1684.

13 Udou T, Ichikawa Y: Characteristics of extracellular nuclease production in Staphylococcus aureus. Microbiol Immunol 1979;23: 679-684.

14 Puyet A, Greenberg B, Lacks SA: Genetic and structural characterization of end A. A membrane-bound nuclease required for transformation of Streptococcus pneumoniae. J Mol Biol 1990;213:727-738.

15 Wartha F, Beiter K, Albiger B, Fernebro J, Zychlinsky A, Normark S, Henriques-Normark B: Capsule and D-alanylated lipoteichoic acids protect Streptococcus pneumoniae against neutrophil extracellular traps. Cell Microbiol 2007;9:1162-1171.

16 Lippolis JD, Reinhardt TA, Goff JP, Horst RL: Neutrophil extracellular trap formation by bovine neutrophils is not inhibited by milk. Vet Immunol Immunopathol 2006; 113:248-255.

17 Mohan C, Adams S, Stanik V, Datta SK: Nucleosome: a major immunogen for pathogenic autoantibody-inducing T cells of lupus. J Exp Med 1993;177:1367-1383.
18 Redman CW, Sargent IL: Latest advances in understanding preeclampsia. Science 2005; 308:1592-1594.

19 Clark P, Boswell F, Greer IA: The neutrophil and preeclampsia. Semin Reprod Endocrinol 1998;16:57-64.

20 Zhong XY, Laivuori H, Livingston JC, Ylikorkala O, Sibai BM, Holzgreve W, Hahn $S$ : Elevation of both maternal and fetal extracellular circulating deoxyribonucleic acid concentrations in the plasma of pregnant women with preeclampsia. Am J Obstet Gynecol 2001;184:414-419.

-21 Gupta AK, Hasler P, Holzgreve W, Hahn S: Neutrophil NETs: a novel contributor to preeclampsia-associated placental hypoxia? Semin Immunopathol 2007;29:163-167.

-22 Clark SR, Ma AC, Tavener SA, McDonald B, Goodarzi Z, Kelly MM, Patel KD, Chakrabarti S, McAvoy E, Sinclair GD, Keys EM, Allen-Vercoe E, Devinney R, Doig CJ, Green FH, Kubes P: Platelet TLR4 activates neutrophil extracellular traps to ensnare bacteria in septic blood. Nat Med 2007;13:463-469.

23 von Köckritz-Blickwede M, Goldmann O, Thulin P, Heinemann K, Norrby-Teglund A, Rohde M, Medina E: Phagocytosis-independent antimicrobial activity of mast cells by means of extracellular trap formation. Blood 2008;111:3070-3080.

24 Mekori YA, Metcalfe DD: Mast cells in innate immunity. Immunol Rev 2000;173:131140.

25 Marshall JS: Mast-cell responses to pathogens. Nat Rev Immunol 2004;4:787-799.

26 Malaviya R, Ikeda T, Ross E, Abraham SN: Mast cell modulation of neutrophil influx and bacterial clearance at sites of infection through TNF- $\alpha$. Nature 1996;381:77-80. 